\title{
Editorial
}

\section{Reviewer acknowledgment 2017}

\section{To Our Reviewers}

The Editor"s of Neurologia i Neurochirurgia Polska (Polish Journal of Neurology and Neurosurgery) wish to thank all members of Polish and International academic community who participated in the process of reviewing articles for our journal in the last year. Thank you very much for your valuable time, knowledge, and expertise. We very much hope that you will further support our efforts of making this journal even better platform for future scientific and educational reports.

List of Reviewers for 2017:

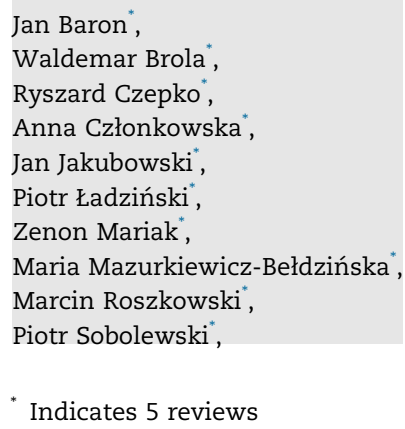

Sumit Dua, Joseph Duffy, Tomasz Dziedzic, Andrzej Friedman, Tatsuo Fuchigami, Agata Gajos, Dariusz Gąsecki, Aysegül Güler, Jerzy Gosk, Marek Harat, Lukas Heydrich, Pieter Hoekstra, Michal Harciarek, Tomas Holubec, Satoshi Hori, Lijia Huang, Iwona Hus, Sung Ho Jang, Esma Isenovic, Dariusz Jaskólski, Maciej Juryńczyk, Anna Jamroz-Wiśniewska, Piotr Jankowski, Roman Jankowski, Krystyna Jaracz, Maria Jędrzejowska, Sergiusz Jóźwiak, Hiroharu Kataoka, Jan Keppel Hesselink, Jiri Klempir, Norito Kokubun, Grzegorz Kopeć, Magdalena Koszewicz, Kaarina Kowalec, Dariusz Koziorowski, Jera Kruja,

Anna Krygowska-Wajs, Alina Kułakowska, Anna Kamińska, Bartosz Karaszewski, Wojciech Kozubski, Radosław Kaźmierski, Agnieszka Korfel, Przemysław Kunert, Angelo Labate, Dawid Larysz, Da Zhi Liu, Tomasz Łysoń, Francesco Latini, Dariusz Łatka, 
Marta Leńska-Mieciek,

Song Liu,

Maria Łukasik,

Tomasz Mandat,

Michael Mlynash,

Hiroyuki Murai,

Marek Mandera,

Domenico Murrone,

Andrew Maas,

Agata Majos,

Sara Mariotto,

Negin Masoudi Alavi,

Nikolaus Mcfarland,

Raja Mehanna,

Jan Mejnartowicz,

Grzegorz Miękisiak,

Dagmara Mirowska-Guzel,

Raffaella Mormile,

Rouzbeh Motiei-Langroudi,

Anastasios Mpotsaris,

Adam Niezgoda,

Monika Nojszewska,

Józef Opara,

Fabrizio Piazza,

Kwangyeol Park,

Traian Popa,

Ji Hoon Phi,

Anna Przeklasa-Muszyńska,

Przemysław Puż,

Natalia Pérez de la Ossa,

Dimitri Renard,

Riccardo Antonio Ricciuti,

Radosław Rola,

David Z. Rose,

Guy Rosenthal,

Konrad Rejdak,

Mayela Rodriguez-Violante,

Alexander Rossor,

Jacek Różniecki,

Małgorzata Rutkiewicz-Hanczewska,

Nishanth Sadashiva,

Valentina Saia,

Kenji Sakai,

Paul Siba

Filippo Santorelli,

Iwona Sarzyńska-Długosz,

Gabriella Silvestri,

Guy Simonnet,

Antonio Siniscalchi,

Agnieszka Słowik,

Emilian Snarski,

Pawel Sokal,

Chrysoula Spanou,

Jacek Staszewski,
Barbara Steinborn,

Vadym Sulyma,

Małgorzata Szczerbo-Trojanowska,

Marcin Szymański,

Maciej Szajner

Mariacristina Scoto,

Andreas Straube,

Clare Thetford,

Claudius Thome,

Marcin Tyrakowski,

Armando Tartaro,

Marcin Tutaj,

Tomasz Trojanowski,

Ekrem Unal,

Vladimira Vuletic,

Vitor Valenti,

Gönül Vural,

László Vécsei,

Jerzy Walecki

Zhanxiang Wang,

Ida Wiszomirska,

Grzegorz Witkowski,

Zbigniew Wszołek,

Guofeng Wu,

Kaan Yağmurlu,

Shams Y-Hassan,

Jun Yang,

An-An Yin,

Xiang Yan Chen,

Jacek Zaborski,

Daniel Zielonka,

Natalie Zahr,

Beata Zakrzewska-Pniewska,

Krzysztof Zapałowicz,

Baron Zarate-Kalfópulos,

" Indicates 5 reviews

Zbigniew K. Wszołek M.D., Co-Editor-in-Chief Jarosław Sławek M.D., Ph.D., Co-Editor-in-Chief Mariusz Sieminski M.D., Ph.D. Technical Editor

E-mail address: msiem@wp.pl (M. Sieminski)

Available online 13 April 2018

https://doi.org/10.1016/j.pjnns.2018.04.001

0028-3843/

(C) 2018 Published by Elsevier Sp. z o.o. on behalf of Polish Neurological Society. 